\title{
Three new species of Saccharomyces sensu lato van der Walt from Yaku Island in Japan: Saccharomyces naganishii sp. nov., Saccharomyces humaticus sp. nov. and Saccharomyces yakushimaensis sp. nov.
}

\author{
1 Institute for Fermentation, \\ Osaka (IFO) 17-85, Juso- \\ honmachi 2-chome, \\ Yodogawa-ku, Osaka \\ 532-8686, Japan \\ 2 Department of \\ Biotechnology, Faculty of \\ Engineering, Fukuyama \\ University 1-3, Gakuen- \\ cho, Fukuyama, Hiroshima \\ 729-0292, Japan
}

\author{
Kozaburo Mikata, ${ }^{1}$ Kumiko Ueda-Nishimura ${ }^{1}$ and Taisuke Hisatomi ${ }^{2}$ \\ Author for correspondence: Kumiko Ueda-Nishimura. Tel: +8166300 6555. Fax: +81663006814. \\ e-mail: nishimura-kumiko@ifo.or.jp
}

Three new yeast species were isolated from soil and partially decayed leaves in Yaku Island and Iriomote Island in the Nansei Islands of Japan. Based on DNA hybridization and physiological characters, these represent novel taxa. These are designated Saccharomyces naganishii sp. nov. (type strain IFO $10181^{\top}=$ CBS $8^{8797^{\top}}$ ), Saccharomyces humaticus sp. nov. (type strain IFO $10673^{\top}={\text { CBS } 8893^{\top} \text { ) }}^{\top}$ and Saccharomyces yakushimaensis sp. nov. (type strain IFO $1889^{\top}=$ CBS $8894^{\top}$ ). The phylogenetic relationships of the three new species with members of other ascomycetous genera (e.g. Kluyveromyces, Saccharomyces, Torulaspora and Zygosaccharomyces) were estimated by 185 rDNA gene sequence analysis.

Keywords: Saccharomyces naganishii sp. nov., Saccharomyces humaticus sp. nov., Saccharomyces yakushimaensis sp. nov., DNA-DNA hybridization, electrophoretic karyotype

\section{INTRODUCTION}

The generic name Saccharomyces was introduced by Meyen (1838) and defined by Reess (1870). In the 4th edition of The Yeasts, a Taxonomic Study, 14 species (Saccharomyces barnettii, Saccharomyces bayanus, Saccharomyces castellii, Saccharomyces cerevisiae, Saccharomyces dairenensis, Saccharomyces exiguus, Saccharomyces kluyveri, Saccharomyces paradoxus, Saccharomyces pastorianus, Saccharomyces rosinii, Saccharomyces servazzii, Saccharomyces spencerorum, Saccharomyces transvaalensis and Saccharomyces unisporus) were described for the genus (Vaughan-Martini \& Martini, 1998). James et al. (1997) analysed relationships among Saccharomyces species based on $18 \mathrm{~S}$ rDNA sequences and described two new species, Saccharomyces kunashirensis and Saccharomyces martiniae; while Wyder et al. (1999) recently described the new species Saccharomyces turicensis isolated from kefyr.

The GenBank/EMBL/DDBJ accession numbers sequences are AB016512 (IFO $10181^{\top}$ ), AB016513 (IFO 10673') and AB016514 (IFO 1889') for the 18S rDNA sequences and AB040999 (IFO 10673') and AB041000 (IFO 1889') for the $26 \mathrm{~S}$ rDNA domain D1/D2 sequences.
In this study, it is shown that nine isolates from Japan represent three new species of Saccharomyces sensu lato. These are proposed as Saccharomyces naganishii, Saccharomyces humaticus and Saccharomyces yakushimaensis.

\section{METHODS}

Yeast strains and identification. The procedure used for yeast isolation and purification has been described previously (Banno \& Mikata, 1981). The strains isolated are listed in Table 1. Saccharomyces type strains used for reference were obtained from the culture collection of the Institute for Fermentation, Osaka (IFO), Japan. Physiological, morphological and cultural characteristics were investigated according to the methods of Yarrow (1998).

Major ubiquinone analysis. The yeast strains were grown at $28^{\circ} \mathrm{C}$ with shaking for $2 \mathrm{~d}$ in 11 Erlenmeyer flasks containing $400 \mathrm{ml}$ YPD broth (1\% Bacto-yeast extract, 2\% Bactopeptone and $2 \%$ glucose). The extraction, purification and analysis of ubiquinone were performed as described by Mikata \& Yamada (1999).

DNA preparation. The yeast strains were grown at $28^{\circ} \mathrm{C}$ with shaking for 10-24 h in $200 \mathrm{ml}$ Erlenmeyer flasks containing $50 \mathrm{ml}$ of YPD broth. Protoplast preparation and DNA extraction followed the protocol of Holm et al. (1986) as modified by Kaneko \& Banno (1991). 
Table 1. Isolated yeast strains

\begin{tabular}{|c|c|c|c|}
\hline \multicolumn{2}{|c|}{ Strain } & \multirow[t]{2}{*}{ Sample material } & \multirow[t]{2}{*}{ Locality (Japan) } \\
\hline Original no. & IFO no. & & \\
\hline Yp74L-3 $3^{\mathrm{T}}$ & $10181^{\mathrm{T}}$ & Decayed leaf & Yaku Island \\
\hline Yp20d & 10922 & Decayed leaf & Yaku Island \\
\hline Ys63a & 10923 & Soil & Yaku Island \\
\hline U97-433-1 & 10924 & Leaf of Kandelia candel & Iriomote Island \\
\hline Ys $79 a^{T}$ & $10673^{\mathrm{T}}$ & Soil & Yaku Island \\
\hline Yp53c- $2^{\mathrm{T}}$ & $1889^{\mathrm{T}}$ & Decayed leaf & Yaku Island \\
\hline Yp3a1 & 10674 & Decayed leaf & Yaku Island \\
\hline Yp31b & 10675 & Decayed leaf & Yaku Island \\
\hline Ys21b & 10676 & Soil & Yaku Island \\
\hline
\end{tabular}

DNA $\mathbf{G}+\mathbf{C}$ determination. The nuclear DNA base compositions were determined by HPLC as described by Tamaoka \& Komagata (1984).

DNA-DNA hybridization. DNA-DNA base sequence similarity between strains was studied by the photobiotin microplate-hybridization method of Ezaki et al. $(1988,1989)$ as modified by Kaneko \& Banno (1991).

PFGE. Yeast cells were harvested from $5 \mathrm{ml}$ of a culture grown overnight in YPD broth. Agarose blocks containing chromosomal DNA were prepared according to the method of Carle \& Olson (1984). Electrophoresis was carried out following the method of Ueda-Nishimura \& Mikata (1999). DNA bands stained in ethidium bromide were observed on a transilluminator (TL-33; Ultra-Violet Products) and photographed.

18S rRNA gene sequencing and phylogenetic analysis. 18S rRNA gene (rDNA) sequences were determined using a Thermo Sequenase fluorescent labelled primer cycle sequencing kit with 7-deaza-dGTP (Amersham Pharmacia Biotech) following the supplier's protocol. PCR for amplification of $18 \mathrm{~S}$ rDNA and cycle sequencing using PCR products were performed as described by Ueda-Nishimura \& Mikata (1999).

Sequence data were aligned manually with various $18 \mathrm{~S}$ rDNA sequences for representatives of related genera obtained from the GenBank. A phylogenetic tree was constructed by Kimura's two-parameter method (Kimura, 1980) and the neighbour-joining method (Saitou \& Nei, 1987) using CLUSTAL W. Bootstrap values (Felsenstein, 1985) were calculated from 1000 replicates.

Domain D1/D2 26S rRNA gene sequencing. The 600 nucleotide 26S rDNA domain D1/D2 sequences were determined by using a BigDye Terminator Cycle Sequencing FS Ready Reaction Kit (PE Biosystems), CentriSep spin column (PE Biosystems) and the ABI PRISM 310 Genetic Analyzer (PE Biosystems) following the manufacturer's protocol. For amplification of domain D1/D2 26S rDNA sequences, PCR (Saiki et al., 1988) was performed for 30 cycles of denaturation at $94^{\circ} \mathrm{C}$ for $30 \mathrm{~s}$, annealing at $57^{\circ} \mathrm{C}$ for $30 \mathrm{~s}$, and extension at $72{ }^{\circ} \mathrm{C}$ for $45 \mathrm{~s}$ with TaKaRa Taq DNA polymerase (Takara) using the primer pair NL-1 (5'-GCA TAT CAA TAA GCG GAG GAA AAG-3') and NL-4 (5'GGT CCG TGT TTC AAG ACG G-3') (Kurtzman \&
Robnett, 1997). The PCR products were purified with GFX PCR DNA and Gel Band Purification Kit (Amersham Pharmacia Biotech) following the supplier's protocol. For sequencing, purified PCR products were used as a template and NL-1 and NL-4 were used as primers.

\section{RESULTS AND DISCUSSION}

\section{Morphology and major ubiquinone of isolated strains}

In all isolates, persistent asci arose directly from diploid cells, forming 1-4 globose to subglobose ascospores (Fig. 1, lower). As monosporic cultures from four-spored asci could sporulate, they were judged to be homothallic. The major ubiquinone of all nine isolates was Q-6. These results suggested that all isolates might be put in the genus Saccharomyces.

\section{Electrophoretic karyotypes}

Electrophoretic karyotyping has been used as a tool for the distinction of species in the genus Saccharomyces (Naumov et al., 1995; Naumova et al., 1996; Vaughan-Martini, 1995; Vaughan-Martini \& Barcaccia, 1996; Vaughan-Martini et al., 1993, 1996). The nine isolates were categorized into three groups based on their electrophoretic karyotypes, which differed from those of the type strains for all currently described Saccharomyces species (data not shown). The karyotype patterns of IFO $10181^{\mathrm{T}}$, IFO 10922 , IFO 10923 and IFO 10924 were similar to each other and displayed 12 bands in a wide range of sizes $(0 \cdot 5-2 \cdot 2$ Mb) (Fig. 2, lane 2). That of IFO $10673^{\mathrm{T}}$ was unique having 10 bands ranging from 0.5 to $2 \cdot 2 \mathrm{Mb}$ (Fig. 2, lane 3). The patterns of IFO $1889^{\mathrm{T}}$, IFO 10674, IFO 10675 and IFO 10676 were similar and displayed 10 bands in a wide range of sizes $(0 \cdot 4-2 \cdot 2 \mathrm{Mb})$ (Fig. 2, lane 4). As a consequence, when strain IFO $10181^{\mathrm{T}}$, initially classified as $S$. exiguus based on its physiological characteristics, showed a chromosomal pattern significantly different from that species, it was listed as 

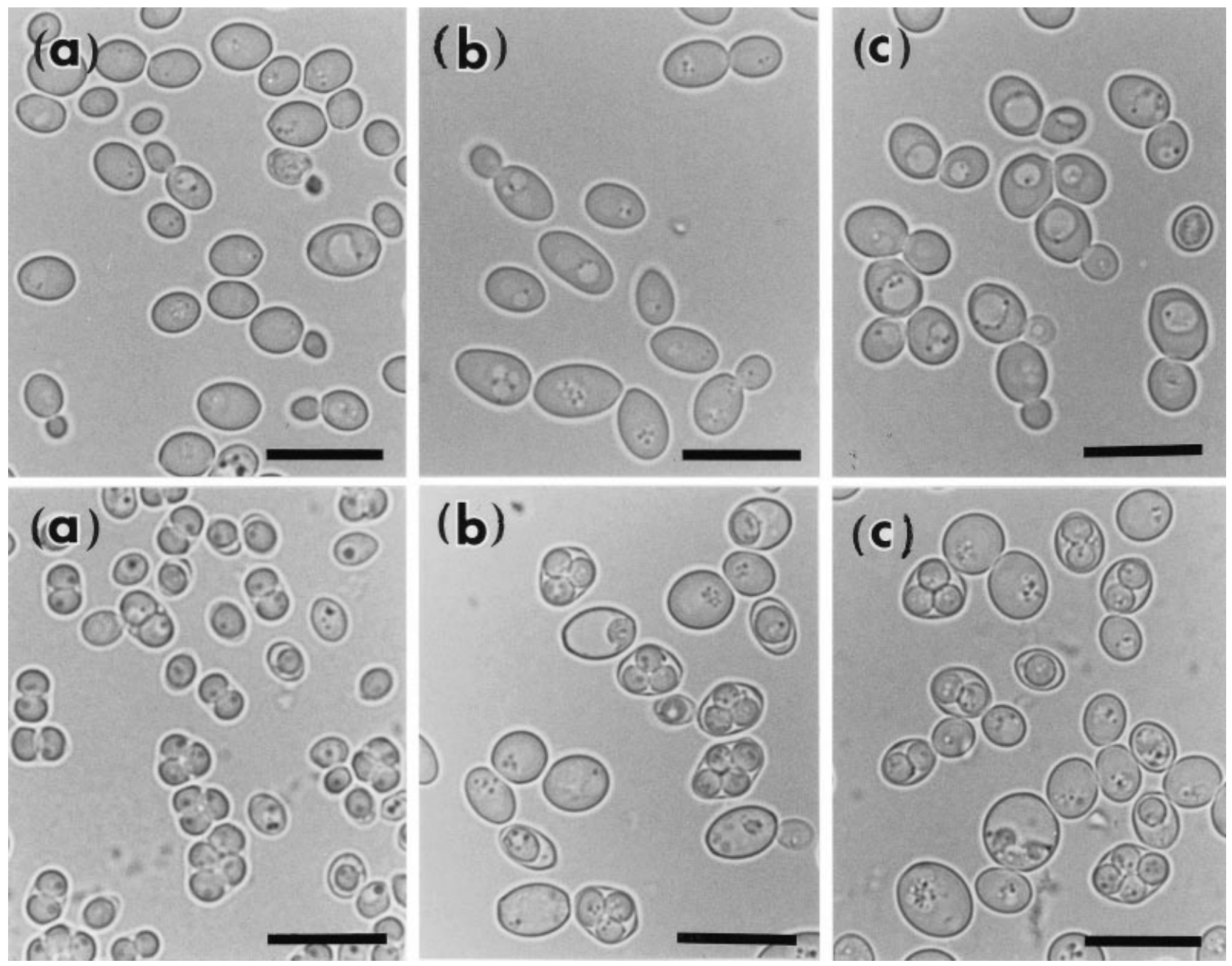

Fig. 1. Photomicrographs of vegetative cells grown on $Y M$ broth for $3 \mathrm{~d}$ at $24{ }^{\circ} \mathrm{C}$ (upper) and sporulated cells on potassium acetate agar for $7 \mathrm{~d}$ at $24^{\circ} \mathrm{C}$ (lower). (a) S. naganishii IFO 10181'; (b) S. humaticus IFO $10673^{\top}$; (c) S. yakushimaensis IFO $1889^{\top}$. Bars, $10 \mu \mathrm{m}$.

Saccharomyces sp. in the 10th edition of the IFO list of cultures (1996). In addition, all of the new species had karyotypes sufficiently different from those of $S$. cerevisiae and related species to indicate that the isolates did not belong to the Saccharomyces sensu stricto complex (Vaughan-Martini et al., 1993).

\section{DNA base compositions and DNA-DNA hybridization}

The DNA $\mathrm{G}+\mathrm{C}$ contents of the isolates were $31 \cdot 0$ $31 \cdot 5,33 \cdot 8$ and $44 \cdot 0-44 \cdot 7 \mathrm{~mol} \%$, respectively (Table 2 ). DNA base sequence similarity tested against type strains of Saccharomyces sensu lato species by the microplate-hybridization method showed that IFO $10673^{\mathrm{T}}$ had low similarity to all tested strains (Table 2), while IFO $10181^{\mathrm{T}}$, IFO 10922, IFO 10923 and IFO 10924 are conspecific, with similarity levels ranging from 82.7 to $97 \cdot 2 \%$. Strains IFO $1889^{\mathrm{T}}$, IFO 10674 , IFO 10675 and IFO 10676 also showed high similarity with one another $(88 \cdot 0-97 \cdot 0 \%)$. These were the same groupings revealed by PFGE patterns. A representative strain of each group showed mostly low base sequence similarity $(2 \cdot 4-49 \cdot 7 \%)$ to all other Saccharomyces sensu lato species.

Based on the results of these determinations, three new species are proposed as Saccharomyces naganishii sp. nov., Saccharomyces humaticus sp. nov. and Saccharomyces yakushimaensis sp. nov.

\section{Latin diagnosis of Saccharomyces naganishii Mikata, Ueda-Nishimura et Hisatomi sp. nov.}

In medio liquido $Y M$ post dies 3 ad $24{ }^{\circ} \mathrm{C}$, cellulae singulae aut binae, cellulae globosae vel ellipsoideae $(2.7-6.0) \times(3.5-8.0) \mu \mathrm{m}$. Post 1 mensem sedimentum formatur. In agaro $Y M$ post 1 memsum ad $17^{\circ} \mathrm{C}$, cremea aut suffuscus, glabra, butyrosa, nitida, margine glabra. In agaro farinae Zea mays post dies 7 , pseudohyphae nullae. Asci inconjugatio fiunt. Ascosporae 


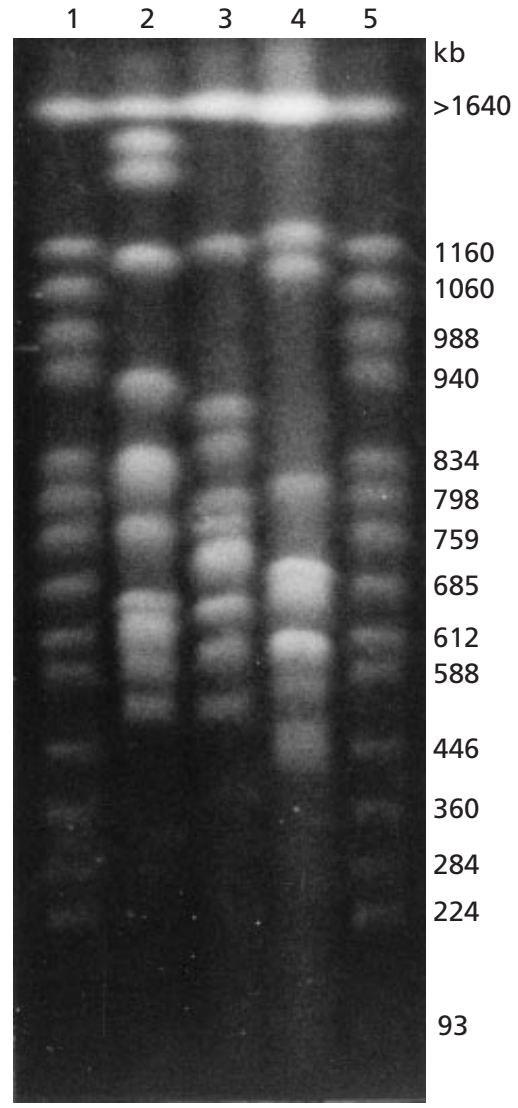

Fig. 2. Electrophoretic karyotypes of Saccharomyces sensu lato. Lanes: 1 \& 5, S. cerevisiae IFO 10613 (size marker); 2, S. naganishii IFO $10181^{\top} ; 3$, S. humaticus IFO $10673^{\top} ; 4$, S. yakushimaensis IFO $1889^{\top}$.

rotundae aut ovoidae $(2 \cdot 3-3 \cdot 8) \times(2 \cdot 3-4 \cdot 2) \mu \mathrm{m}, 1-4$ in quoque asco.

Glucosum, galactosum, sucrosum, trehalosum et raffinosum fermentatur, at non maltosum, lactosum, inulinum nec amylum solubile. Glucosum, galactosum, sucrosum, trehalosum, raffinosum, D-gluconatum, 5ketogluconatum et D-glucono-1,5-lactonum assimilantur, at non L-sorbosum, maltosum, cellobiosum, lactosum, melibiosum, melezitosum, inulinum, amylum solubile, D-xylosum, L-arabinosum, D-arabinosum, D-ribosum, L-rhamnosum, ethanolum, methanolum, glycerolum, erythritolum, ribitolum, galactitolum, Dmannitolum, D-glucitolum, methyl $\alpha$-D-glucosidum, salicinum, acidum DL-lacticum, acidum succinicum, acidum citricum, arbutinum, inositolum, 2-ketogluconatum, glucosaminum, $N$-acetyl-D-glucosaminum nec hexadecanum. Ethylaminum, L-lysinum et cadaverinum assimilantur, at non kalium nitricum. Ad crescentiam vitaminae externae necessariae sunt. Augmentum in $34{ }^{\circ} \mathrm{C}$, raro in $37{ }^{\circ} \mathrm{C}$. In $10 \%$ natrium chloridum $/ 5 \%$ glucoso crescentiae at non $0 \cdot 1 \%$ cycloheximido. Ureum non hydrolysature. Diazonium caeruleum $B$ non respondens. Amylum non formatur. Guaninum et cytosinum acidi deoxyribonucleati 44-45 mol\%. Systema coenzymatis Q-6 adest.
Typus stirps IFO $10181^{T}\left(=\mathrm{CBS} 8797^{\mathrm{T}}\right)$ isolatus ex partim putridus folia, in Yuku insula, Kagoshima Pref., Japonia, VIII 1971, I. Banno, conservatur in collectionibus culturarum quas 'Institute for Fermentation, Osaka (IFO)', sub no. IFO $10181^{\mathrm{T}}$ deposita est.

\section{Description of Saccharomyces naganishii Mikata, Ueda-Nishimura et Hisatomi sp. nov.}

Saccharomyces naganishii (na.ga.ni.shi.i. L. gen. masc. n. naganishii of Naganishi, in honour of Hirosuke Naganishi).

Growth in YM medium: after $3 \mathrm{~d}$ at $24^{\circ} \mathrm{C}$, the cells are globose to ellipsoidal $(2 \cdot 7-6 \cdot 0) \times(3 \cdot 5-8 \cdot 0) \mu \mathrm{m}$, single or in pairs (Fig. 1a, upper). After 1 month at $17^{\circ} \mathrm{C}$, a sediment is present and occasionally a thin film. Growth on YM agar medium: after 1 month at $17^{\circ} \mathrm{C}$ the streak culture is butyrous, cream to slightly brownish, smooth, scanty growth, rather flat or raised, dull, with an entire or undulating margin. Dalmau plate culture on corn meal agar: after $7 \mathrm{~d}$ at $24^{\circ} \mathrm{C}$, pseudohyphae are not formed. Formation of ascospores: vegetative cells are transformed directly into persistent asci containing 1-4 globose to subglobose $(2 \cdot 3-3 \cdot 8) \times(2 \cdot 3-4 \cdot 2) \mu \mathrm{m}$, smooth ascospores (Fig. 1a, lower). Sporulation was observed on acetate agar, corn meal agar and $\mathrm{YM}$ agar after $7 \mathrm{~d}$ incubation at $24{ }^{\circ} \mathrm{C}$.

Glucose, galactose, sucrose, trehalose and raffinose are fermented, but not maltose, lactose, inulin or soluble starch. Glucose, galactose, sucrose, trehalose, raffinose, D-gluconate, 5-ketogluconate and Dglucono-1,5-lactone are assimilated, but not L-sorbose, maltose, cellobiose, lactose, melibiose, melezitose, inulin, soluble starch, D-xylose, L-arabinose, D-arabinose, D-ribose, L-rhamnose, ethanol, methanol, glycerol, erythritol, ribitol, galactitol, D-mannitol, Dglucitol, methyl- $\alpha$-D-glucoside, salicin, DL-lactic acid, succinic acid, citric acid, arbutin, inositol, 2ketogluconate, glucosamine, $N$-acetyl-D-glucosamine or hexadecane. Ethylamine, L-lysine and cadaverine are assimilated as a sole nitrogen sources, but not potassium nitrate. Growth in vitamin free-medium is negative. Growth at $34^{\circ} \mathrm{C}$ is positive, at $37^{\circ} \mathrm{C}$ negative. Growth in the presence of 1000 p.p.m. cycloheximide is negative. Growth on $50 \%(\mathrm{w} / \mathrm{w})$ glucoseyeast extract agar is negative. Growth in $10 \%$ sodium chloride plus $5 \%$ glucose in yeast nitrogen base is positive. Diazonium blue $\mathrm{B}$ reaction is negative. Production of starch-like substances is negative. Urease activity is negative. Ubiquinone type is Q-6. The $\mathrm{G}+\mathrm{C}$ content of the nuclear DNA is $44-45$ $\mathrm{mol} \%$.

Strains IFO $10181^{\mathrm{T}}$ and IFO 10922 from partially decayed leaves and IFO 10923, from soil were isolated in August 1971 in Yaku Island, Japan. IFO 10924 was isolated in July 1997 from a leaf of Kandelia candel in Iriomote Island, Japan. Cultures of the type strain IFO $10181^{\mathrm{T}}\left(=\mathrm{CBS} 8797^{\mathrm{T}}\right)$ have been deposited in the 
Table 2. G + C content and DNA base sequence similarity between the new species and others of Saccharomyces

Bold numbers indicate similarity values for the same species.

\begin{tabular}{|c|c|c|c|c|}
\hline \multirow[t]{2}{*}{ Strain } & \multirow[t]{2}{*}{$\mathbf{G}+\mathbf{C}(\mathbf{m o l} \%)$} & \multicolumn{3}{|c|}{ DNA similarity (\%) } \\
\hline & & IFO $10181^{\mathrm{T}}$ & IFO $10673^{T}$ & IFO $1889^{\mathrm{T}}$ \\
\hline \multicolumn{5}{|l|}{ S. yakushimaensis } \\
\hline IFO 10674 & ND & ND & ND & $97 \cdot 0$ \\
\hline IFO $1889^{\mathrm{T}}$ & $31 \cdot 0$ & $26 \cdot 1$ & $30 \cdot 5$ & 100 \\
\hline IFO 10676 & $31 \cdot 3$ & ND & $35 \cdot 0$ & $90 \cdot 5$ \\
\hline IFO 10675 & $31 \cdot 5$ & ND & ND & 88.0 \\
\hline S. unisporus IFO $0316^{\mathrm{T}}$ & $32 \cdot 3$ & $2 \cdot 4$ & $19 \cdot 9$ & $25 \cdot 0$ \\
\hline S. exiguus IFO $1128^{\mathrm{T}}$ & $32 \cdot 5$ & $9 \cdot 2$ & $19 \cdot 8$ & $23 \cdot 0$ \\
\hline S. transvaalensis IFO $1625^{\mathrm{T}}$ & $33 \cdot 1$ & $26 \cdot 5$ & $49 \cdot 7$ & $33 \cdot 1$ \\
\hline S. spencerorum IFO $10851^{\mathrm{T}}$ & $33 \cdot 5$ & $22 \cdot 4$ & $21 \cdot 5$ & $26 \cdot 6$ \\
\hline S. kunashirensis IFO $10915^{\mathrm{T}}$ & $33 \cdot 5$ & $4 \cdot 0$ & $5 \cdot 0$ & $2 \cdot 4$ \\
\hline S. barnettii IFO $10849^{\mathrm{T}}$ & $33 \cdot 7$ & $21 \cdot 9$ & $24 \cdot 0$ & $29 \cdot 1$ \\
\hline S. humaticus IFO $10673^{\mathrm{T}}$ & $33 \cdot 8$ & $17 \cdot 9$ & 100 & $38 \cdot 7$ \\
\hline S. dairenensis IFO $0211^{\mathrm{T}}$ & $34 \cdot 1$ & $27 \cdot 0$ & $15 \cdot 6$ & $10 \cdot 7$ \\
\hline S. servazzii IFO $1838^{\mathrm{T}}$ & $34 \cdot 4$ & $30 \cdot 4$ & $16 \cdot 6$ & $15 \cdot 9$ \\
\hline S. martiniae IFO $0752^{\mathrm{T}}$ & $35 \cdot 2$ & $24 \cdot 6$ & $23 \cdot 3$ & $36 \cdot 5$ \\
\hline S. castellii IFO $1992^{\mathrm{T}}$ & $35 \cdot 3$ & $31 \cdot 8$ & $20 \cdot 3$ & $21 \cdot 5$ \\
\hline S. cerevisiae IFO $10217^{\mathrm{T}}$ & $38 \cdot 0$ & $25 \cdot 5$ & $29 \cdot 4$ & $38 \cdot 4$ \\
\hline S. pastorianus IFO 1167 & $38 \cdot 3$ & ND & ND & ND \\
\hline S. paradoxus IFO $10609^{\mathrm{T}}$ & $38 \cdot 6$ & ND & ND & ND \\
\hline S. bayanus IFO $1127^{\mathrm{T}}$ & $39 \cdot 0$ & ND & ND & ND \\
\hline S. rosinii IFO $10008^{\mathrm{T}}$ & $39 \cdot 7$ & $18 \cdot 3$ & $19 \cdot 9$ & $25 \cdot 1$ \\
\hline S. kluyveri IFO $1685^{\mathrm{T}}$ & $40 \cdot 0$ & $14 \cdot 7$ & $15 \cdot 6$ & $20 \cdot 6$ \\
\hline \multicolumn{5}{|l|}{ S. naganishii } \\
\hline IFO 10924 & $44 \cdot 0$ & $82 \cdot 7$ & ND & ND \\
\hline IFO 10922 & $44 \cdot 5$ & $97 \cdot 2$ & ND & ND \\
\hline IFO $10181^{\mathrm{T}}$ & $44 \cdot 6$ & 100 & ND & ND \\
\hline IFO 10923 & $44 \cdot 7$ & 83.9 & ND & ND \\
\hline
\end{tabular}

ND, Not determined.

culture collection of the Institute for Fermentation, Osaka (IFO).

\section{Latin diagnosis of Saccharomyces humaticus Mikata et Ueda-Nishimura sp. nov.}

In medio liquido $Y M$ post dies 3 ad $24{ }^{\circ} \mathrm{C}$, cellulae singulae aut binae, cellulae globosae vel ellipsoideae $(4 \cdot 3-6 \cdot 4) \times(5 \cdot 9-11 \cdot 0) \mu \mathrm{m}$. Post $1 \mathrm{mensem}$ sedimentum formatur. In agaro $Y M$ post 1 memsum ad $17^{\circ} \mathrm{C}$, cremea, glabra, butyrosa, nitida, margine glabra. In agaro farinae Zea mays post dies 7, primitivus pseudomycelium formantur. Asci inconjugatio fiunt. Ascosporae rotundae aut ovoidae $(2 \cdot 7-4 \cdot 3) \times(2 \cdot 7-4 \cdot 3) \mu \mathrm{m}$, 1-4 in quoque asco.

Glucosum et galactosum fermentatur, at non sucrosum, maltosum, trehalosum, lactosum, raffinosum, inulinum nec amylum solubile. Glucosum et galactosum assimilantur, at non L-sorbosum, sucrosum, maltosum, cellobiosum, trehalosum, lactosum, melibiosum, raffinosum, melezitosum, inulinum, amylum solubile, D-xylosum, Larabinosum, D-arabinosum, D-ribosum, L-rhamnosum, ethanolum, methanolum, glycerolum, erythritolum, ribitolum, galactitolum, D-mannitolum, D-glucitolum, methyl $\alpha$-D-glucosidum, salicinum, acidum DL-lacticum, acidum succinicum, acidum citricum, D-gluconatum, arbutinum, inositolum, 2-ketogluconatum, 5-ketogluconatum, glucosaminum, glucono-1,5-lactonum, $\mathrm{N}$ acetyl-D-glucosaminum nec hexadecanum. Ethylaminum et cadaverinum assimilantur, at non kalium nitricum nec L-lysinum. Ad crescentiam vitaminae externae necessariae sunt. Augmentum in $34{ }^{\circ} \mathrm{C}$, raro in $37^{\circ} \mathrm{C}$. Non crescere potest in $10 \%$ natrium chloridum/ $5 \%$ glucoso nec $0.1 \%$ cycloheximido. Ureum non hydrolysature. Diazonium caeruleum B non respondens. Amylum non formatur. Guaninum et cytosinum acidi deoxyribonucleati $34 \mathrm{~mol} \%$. Systema coenzymatis Q-6 adest.

Typus stirps IFO $10673^{T}\left(=\mathrm{CBS} 8893^{\mathrm{T}}\right)$ isolatus ex terra, in Yuku insula, Kagoshima Pref., Japonia, VIII 1971, I. Banno, conservatur in collectionibus culturarum quas 'Institute for Fermentation, Osaka (IFO)', sub no. IFO $10673^{\mathrm{T}}$ deposita est. 


\section{Description of Saccharomyces humaticus Mikata et Ueda-Nishimura sp. nov.}

Saccharomyces humaticus (hu.ma.ti.cus. L. adj. humaticus the soil from isolated).

Growth in YM medium: after 3 d at $24{ }^{\circ} \mathrm{C}$, the cells are globose to ellipsoidal $(4 \cdot 3-6 \cdot 4) \times(5 \cdot 9-11 \cdot 0) \mu \mathrm{m}$, single or in pairs (Fig. $1 \mathrm{~b}$, upper). After 1 month at $17^{\circ} \mathrm{C}$, a sediment is present. Growth on YM agar medium: after 1 month at $17{ }^{\circ} \mathrm{C}$ the streak culture is butyrous, cream to slightly brownish, smooth, scanty growth, rather flat or raised, dull, with an entire or undulating margin. Dalmau plate culture on corn meal agar: after $7 \mathrm{~d}$ at $24^{\circ} \mathrm{C}$, primitive pseudohyphae are formed. Formation of ascospores: vegetative cells are transformed directly into persistent asci containing 1-4 globose to subglobose $(2 \cdot 7-4 \cdot 3) \times(2 \cdot 7-4 \cdot 3) \mu \mathrm{m}$, smooth ascospores (Fig. 1b, lower). Sporulation was observed on acetate agar, corn meal agar and YM agar after $7 \mathrm{~d}$ incubation at $24{ }^{\circ} \mathrm{C}$.

Glucose and galactose are fermented, but not sucrose, maltose, trehalose, lactose, raffinose, inulin or soluble starch. Glucose and galactose are assimilated, but not L-sorbose, sucrose, maltose, cellobiose, trehalose, lactose, melibiose, raffinose, melezitose, inulin, soluble starch, D-xylose, L-arabinose, D-arabinose, D-ribose, Lrhamnose, ethanol, methanol, glycerol, erythritol, ribitol, galactitol, D-mannitol, D-glucitol, methyl $\alpha$-Dglucoside, salicin, DL-lactic acid, succinic acid, citric acid, D-gluconate, arbutin, inositol, 2-ketogluconate, 5-ketogluconate, glucosamine, glucono-1,5-lactone, $\mathrm{N}$-acetyl-D-glucosamine or hexadecane. Ethylamine and cadaverine are assimilated as a sole nitrogen sources, but not potassium nitrate and L-lysine. Growth in vitamin-free medium is negative. Growth at $34{ }^{\circ} \mathrm{C}$ is positive, at $37^{\circ} \mathrm{C}$ negative. Growth in the presence of 1000 p.p.m. of cycloheximide is negative. Growth on $50 \%(\mathrm{w} / \mathrm{w})$ glucose-yeast extract agar is negative. Growth in $10 \%$ sodium chloride plus $5 \%$ glucose in yeast nitrogen base is negative. Diazonium blue $\mathrm{B}$ reaction is negative. Production of starch-like substances is negative. Urease activity is negative. Ubiquinone type is Q-6. The $\mathrm{G}+\mathrm{C}$ content of the nuclear DNA is $34 \mathrm{~mol} \%$.

The type strain IFO $10673^{\mathrm{T}}$ was isolated in August 1971 from soil in Yaku Island, Japan. Cultures of the type strain IFO $10673^{\mathrm{T}}\left(=\mathrm{CBS} 8893^{\mathrm{T}}\right)$ have been deposited in the culture collection of the Institute for Fermentation, Osaka (IFO).

\section{Latin diagnosis of Saccharomyces yakushimaensis Mikata et Ueda-Nishimura sp. nov.}

In medio liquido $Y M$ post dies 3 ad $24{ }^{\circ} \mathrm{C}$, cellulae singulae aut binae, cellulae globosae vel subglobosae $(4 \cdot 8-7 \cdot 0) \times(5 \cdot 9-9 \cdot 1) \mu \mathrm{m}$. Post 1 mensem sedimentum formatur. In agaro $Y M$ post 1 memsum ad $17^{\circ} \mathrm{C}$, cremea, glabra, butyrosa, nitida, margine glabra. In agaro farinae Zea mays post dies 7, pseudomycelium nullae. Asci inconjugatio fiunt. Ascosporae rotundae aut ovoidae $(2 \cdot 5-4 \cdot 3) \times(3 \cdot 2-4 \cdot 8) \mu \mathrm{m}, 1-4$ in quoque asco.
Glucosum et galactosum fermentatur, at non sucrosum, maltosum, trehalosum, lactosum, raffinosum, inulinum et amylum solubile. Glucosum, galactosum et trehalosum (tardus) assimilantur, at non L-sorbosum, sucrosum, maltosum, cellobiosum, lactosum, melibiosum, raffinosum, melezitosum, inulinum, amylum solubile, Dxylosum, L-arabinosum, D-arabinosum, D-ribosum, Lrhamnosum, ethanolum, methanolum, glycerolum, erythritolum, ribitolum, galactitolum, D-mannitolum, Dglucitolum, methyl $\alpha$-D-glucosidum, salicinum, acidum DL-lacticum, acidum succinicum, acidum citricum, Dgluconatum, arbutinum, inositolum, 2-ketogluconatum, 5-ketogluconatum, glucosaminum, glucono-1,5-lactonum, $N$-acetyl-D-glucosaminum nec hexadecanum. Ethylaminum et L-lysine assimilantur, at non kalium nitricum nec cadaverinum. Ad crescentiam vitaminae externae necessariae sunt. Augmentum in $34{ }^{\circ} \mathrm{C}$, raro in $37^{\circ} \mathrm{C}$. Non crescere potest in $10 \%$ natrium chloridum/ $5 \%$ glucoso nec $0.1 \%$ cycloheximido. Ureum non hydrolysature. Diazonium caeruleum B non respondens. Amylum non formatur. Guaninum et cytosinum acidi deoxyribonucleati $31-32 \mathrm{~mol} \%$. Systema coenzymatis Q-6 adest.

Typus stirps IFO $1889^{\mathrm{T}}\left(=\mathrm{CBS} 8894^{\mathrm{T}}\right)$ isolatus ex partim putridus folia, in Yuku insula, Kagoshima Pref., Japonia, VIII 1971, I. Banno, conservatur in collectionibus culturarum quas 'Institute for Fermentation, Osaka (IFO)', sub no. IFO $1889^{\mathrm{T}}$ deposita est.

\section{Description of Saccharomyces yakushimaensis Mikata et Ueda-Nishimura sp. nov.}

Saccharomyces yakushimaensis (ya.ku.shi.ma.en'sis L. adj. yakushimaensis pertaining to Yaku Island, Japan, where the yeast was originally isolated).

Growth in YM medium: after $3 \mathrm{~d}$ at $24^{\circ} \mathrm{C}$, the cells are globose to subglobose $(4 \cdot 8-7 \cdot 0) \times(5 \cdot 9-9 \cdot 1) \mu \mathrm{m}$, single or in pairs (Fig. 1c, upper). After 1 month at $17^{\circ} \mathrm{C}$, a sediment is present. Growth on YM agar medium: after 1 month at $17{ }^{\circ} \mathrm{C}$ the streak culture is butyrous, cream to slightly brownish, smooth, scanty growth, rather flat or raised, dull, with an entire or undulating margin. Dalmau plate culture on corn meal agar: after $7 \mathrm{~d}$ at $24{ }^{\circ} \mathrm{C}$, pseudohyphae are not formed. Formation of ascospores: vegetative cells are transformed directly into persistent asci containing 1-4 globose to subglobose $(2 \cdot 5-4 \cdot 3) \times(3 \cdot 2-4 \cdot 8) \mu \mathrm{m}$, smooth ascospores (Fig. 1c, lower). Sporulation was observed on acetate agar, corn meal agar and YM agar after $7 \mathrm{~d}$ incubation at $24{ }^{\circ} \mathrm{C}$.

Glucose and galactose are fermented, but not sucrose, maltose, trehalose, lactose, raffinose, inulin or soluble starch. Glucose, galactose and trehalose (slow) are assimilated, but not L-sorbose, sucrose, maltose, cellobiose, lactose, melibiose, raffinose, melezitose, inulin, soluble starch, D-xylose, L-arabinose, D-arabinose, Dribose, L-rhamnose, ethanol, methanol, glycerol, erythritol, ribitol, galactitol, D-mannitol, D-glucitol, methyl $\alpha$-D-glucoside, salicin, DL-lactic acid, succinic acid, citric acid, D-gluconate, arbutin, inositol, 
Table 3. Key characters of species of Saccharomyces sensu lato

Su, sucrose; Tr, trehalose; Raf, raffinose; Ma, maltose; Eth, ethanol; Gly, glycerol; LA, lactate; SA, succinate; GA, gluconate; Ety, ethylamine; Lys, lysine; Cad, cadaverine; Cyc, resistance to cycloheximide 1000 p.p.m.; 34 and 37 , growth at 34 or $37{ }^{\circ} \mathrm{C}$. + , Positive growth; -, negative; s, slow growth; v, variable; NT, not tested.

\begin{tabular}{|c|c|c|c|c|c|c|c|c|c|c|c|c|c|c|c|c|c|c|}
\hline \multirow[t]{3}{*}{ Species } & \multicolumn{3}{|c|}{ Fermentation } & \multicolumn{12}{|c|}{ Assimilation } & \multicolumn{3}{|c|}{ Growth } \\
\hline & \multirow[b]{2}{*}{ Su } & \multirow[b]{2}{*}{$\mathbf{T r}$} & \multirow[b]{2}{*}{ Raf } & \multicolumn{9}{|c|}{ Carbon } & \multicolumn{3}{|c|}{ Nitrogen } & \multirow[b]{2}{*}{ Сус } & \multirow[b]{2}{*}{34} & \multirow[b]{2}{*}{37} \\
\hline & & & & Su & Мa & $\operatorname{Tr}$ & Raf & Eth & Gly & $\mathbf{L A}$ & $\mathbf{S A}$ & GA & Ety & Lys & Cad & & & \\
\hline S. sensu stricto* & $\mathrm{V}$ & - & + & + & + & $+\mathrm{s}$ & + & + & $\mathrm{v}$ & $\mathrm{V}$ & $\mathrm{V}$ & $\mathrm{v}$ & - & - & - & - & $\mathrm{V}$ & $\mathrm{V}$ \\
\hline S. barnettii & + & $\mathrm{S}$ & $\mathrm{s}$ & + & - & + & + & - & - & + & + & + & - & - & - & - & - & - \\
\hline S. castellii & - & - & - & - & - & $\mathrm{s}$ & - & - & - & - & $\mathrm{V}$ & + & - & - & - & - & + & - \\
\hline S. dairenensis & - & - & - & - & - & + & - & $\mathrm{V}$ & $\mathrm{v}$ & - & s & $\mathrm{v}$ & - & - & - & - & $\mathrm{V}$ & - \\
\hline S. exiguus & + & + & $\mathrm{s}$ & + & - & + & + & $\mathrm{s}$ & - & - & s & - & - & - & - & $\mathrm{V}$ & $\mathrm{V}$ & - \\
\hline S. humaticus & - & - & - & - & - & - & - & - & - & - & - & - & + & - & + & - & + & - \\
\hline S. kluyveri & + & - & + & + & + & + & + & + & $\mathrm{v}$ & + & - & - & + & + & + & - & + & + \\
\hline S. kunashirensis & - & - & - & - & - & + & - & - & + & - & - & - & - & - & - & - & NT & - \\
\hline S. martiniae & - & + & - & - & - & + & - & - & $\mathrm{V}$ & - & - & + & - & - & - & - & NT & - \\
\hline S. naganishii & + & + & + & + & - & + & + & - & - & - & - & + & + & + & + & - & + & - \\
\hline S. rosinii & - & - & - & - & - & - & - & - & - & - & - & - & - & - & - & - & - & - \\
\hline S. servazzii & - & - & - & - & - & + & - & - & + & - & - & - & - & - & - & + & + & + \\
\hline S. spencerorum & $\mathrm{S}$ & + & - & + & - & + & - & - & + & + & $\mathrm{s}$ & + & + & + & + & - & + & + \\
\hline S. transvaalensis & - & - & - & - & - & + & - & $\mathrm{V}$ & $\mathrm{V}$ & - & - & + & - & $\mathrm{V}$ & $\mathrm{V}$ & - & + & + \\
\hline S. turicensis† & + & + & - & + & - & + & - & - & - & - & - & - & - & - & - & - & NT & - \\
\hline S. unisporus & - & - & - & - & - & $\mathrm{V}$ & - & + & - & - & $\mathrm{s}$ & - & + & + & + & + & + & $\mathrm{V}$ \\
\hline S. yakushimaensis & - & - & - & - & - & $\mathrm{s}$ & - & - & - & - & - & - & + & + & - & - & + & - \\
\hline
\end{tabular}

* Saccharomyces sensu stricto: S. cerevisiae, S. pastorianus, S. paradoxus and S. bayanus.

$\dagger$ Data are from Wyder et al. (1999).

2-ketogluconate, 5-ketogluconate, glucosamine, glucono-1,5-lactone, $N$-acetyl-D-glucosamine or hexadecane. Ethylamine and L-lysine are assimilated as a sole nitrogen source, but not potassium nitrate and cadaverine. Growth in vitamin-free medium is negative. Growth at $34{ }^{\circ} \mathrm{C}$ is positive, at $37^{\circ} \mathrm{C}$ negative. Growth in the presence of 1000 p.p.m. of cycloheximide is negative. Growth on $50 \%(\mathrm{w} / \mathrm{w})$ glucoseyeast extract agar is negative. Growth in $10 \%$ sodium chloride plus $5 \%$ glucose in yeast nitrogen base is negative. Diazonium blue $\mathrm{B}$ reaction is negative. Production of starch like substances is negative. Urease activity is negative. Ubiquinone type is Q-6. The $\mathrm{G}+\mathrm{C}$ content of the nuclear DNA is $31-32$ $\mathrm{mol} \%$.

Strains IFO $1889^{\mathrm{T}}$, IFO 10674 and IFO 10675 from partially decayed leaves and IFO 10676, from soil were isolated in August 1971 in Yaku Island, Japan. Cultures of the type strain IFO $1889^{\mathrm{T}}\left(=\mathrm{CBS} 8894^{\mathrm{T}}\right)$ have been deposited in the culture collection of the Institute for Fermentation, Osaka (IFO).

\section{Physiology}

Differential physiological characteristics for the isolates and other Saccharomyces species are listed in Table 3. Most physiological analysis corresponded to those of previous studies except for the ability of $S$. kunashirensis CBS $7662^{\mathrm{T}}\left(=\mathrm{IFO} 10915^{\mathrm{T}}\right)$ and $S$. martiniae CBS $6334^{\mathrm{T}}\left(=\mathrm{IFO} 0752^{\mathrm{T}}\right)$ to utilize and ferment sucrose. Contrary to others who found that these could ferment but not utilize this suger aerobically as a sole carbon source (James et al., 1997), in this study both tests gave negative results. This seems more in keeping with the fact that the 4th edition of The Yeasts, a Taxonomic Study (Kurtzman \& Fell, 1998) does not list strains able to ferment a compound that it is not able to also use aerobically.

S. naganishii was similar to $S$. barnettii and S. exiguus in its ability to ferment glucose, galactose, sucrose, trehalose and raffinose. However, S. naganishii could be distinguished from S. barnettii and S. exiguus by the assimilation of ethylamine, lysine and cadaverine. $S$. humaticus and $S$. yakushimaensis were similar to each other and to $S$. castellii, S. dairenensis, S. kunashirensis, $S$. rosinii, $S$. servazzii, $S$. transvaalensis and $S$. unisporus in their ability to ferment and assimilate glucose and galactose and their failure to utilize most other compounds. However, S. humaticus and $S$. yakushimaensis could be distinguished from the above species by the combination of ethylamine, lysine and cadaverine assimilation. Furthermore, they can be distinguished by trehalose, lysine and cadaverine assimilations and $\mathrm{mol} \% \mathrm{G}+\mathrm{C}$ contents, although 


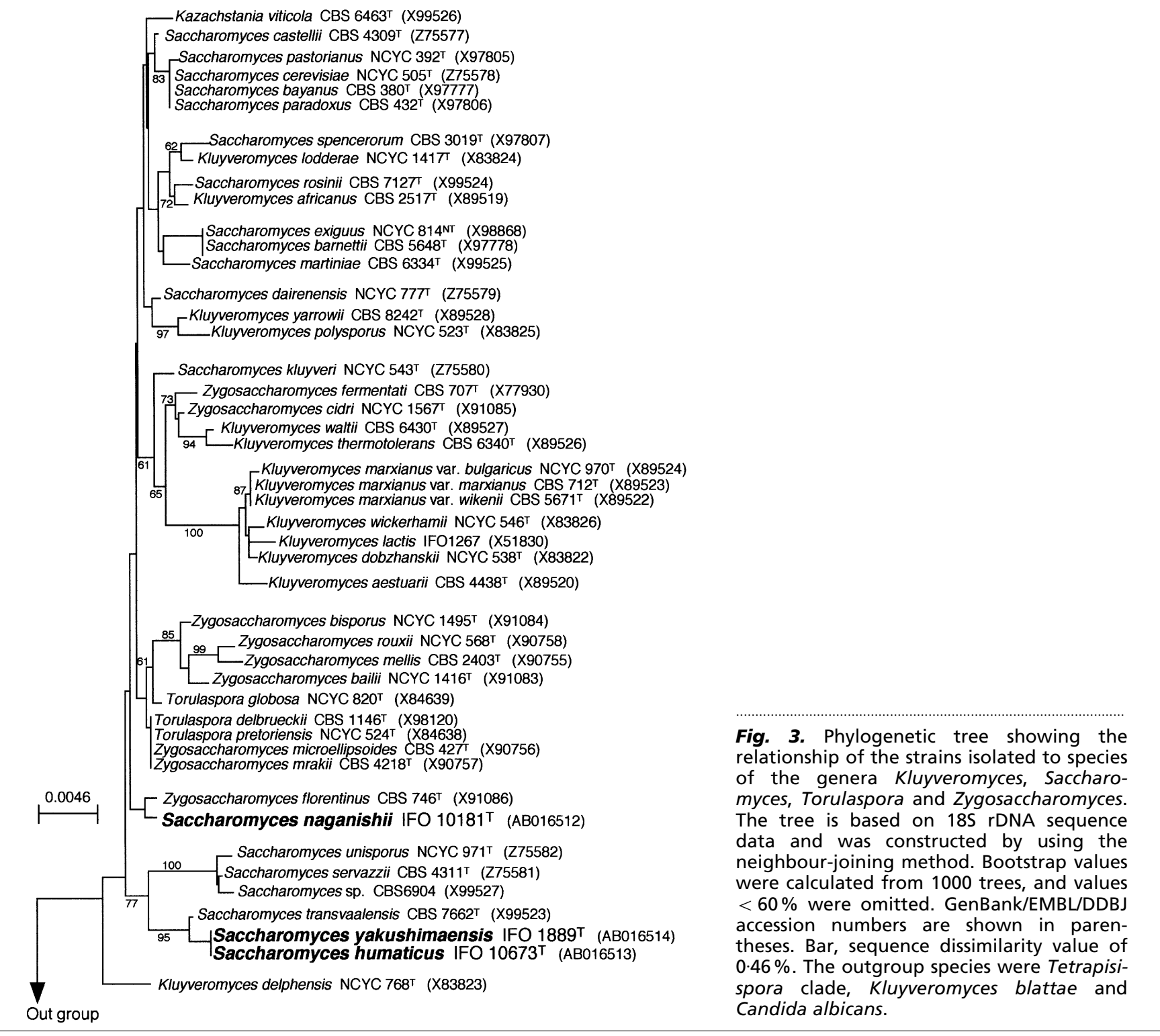

most other physiological characters were similar (Table 3).

\section{Phylogeny}

18S rDNA sequences of $S$. naganishii IFO $10181^{\mathrm{T}}, S$. humaticus IFO $10673^{\mathrm{T}}$ and $S$. yakushimaensis IFO $1889^{\mathrm{T}}$ were determined by the direct method, and a phylogenetic tree was generated by the neighbourjoining method (Fig. 3).

S. naganishii IFO $10181^{\mathrm{T}}$ displayed no specific affinity with any other species of Saccharomyces. However, this strain and Zygosaccharomyces florentinus form a cluster at a $45 \%$ bootstrap confidence level (omitted from Fig. 3). Phylogenetic analyses by James et al. $(1994,1997)$ showed that $Z$. florentinus forms a distinct line, showing no specific affinity with any other Zygosaccharomyces, Saccharomyces, Kluyveromyces or Torulaspora. In addition, the $\mathrm{G}+\mathrm{C}$ content of $S$. naganishii (44.0-44.7 mol\%), was higher than any other species of Saccharomyces (Table 2), while being similar to that of $Z$. florentinus $(42 \cdot 4-43 \cdot 1 \mathrm{~mol} \%)$ (Kurtzman, 1998). Nevertheless, since the bootstrap value supporting the joining of $S$. naganishii and $Z$. florentinus was low (45\%), suggesting that the topology was variable, together with the fact that $S$. naganishii has a Q-6-type system and forms persistent asci, this new species will be temporarily put in the genus Saccharomyces. By the interspecific sexual reactions, it was suggested that $S$. naganishii has some relation to $S$. cerevisiae (Hisatomi et al., 1988). Hisatomi et al. (1986) isolated heterothallic strains from the original homothallic strain $S$. naganishii IFO $10181^{\mathrm{T}}$ by introducing a mutation into a homothallism gene. An a mating-type strain THE1-16C (= IFO 10945) and an $\alpha$ mating-type strain THE1-16B (= IFO 10944) derived from $S$. naganishii IFO $10181^{\mathrm{T}} \mathrm{did}$ not sexually agglutinate or mate with either mating- 
type strain of $S$. cerevisiae, in spite of interspecifically sexual reactions that both $\alpha$ mating pheromones from S. cerevisiae and S. naganishii induce G1-arrest in both a mating-type cells of these two species (Hisatomi et al., 1988).

S. humaticus IFO $10673^{\mathrm{T}}$ and S. yakushimaensis IFO $1889^{\mathrm{T}}$ are found to be closely related to each other (Fig. 3). Although S. humaticus IFO $10673^{\mathrm{T}}$ and $S$. yakushimaensis IFO $1889^{\mathrm{T}}$ were different species on the basis of low DNA reassociation $(30 \cdot 5-38 \cdot 7 \%)$ (Table 2), it was not possible to separate them on the basis of this analysis, the only difference being one base insertion in S. yakushimaensis. As a result, to distinguish these species, their 26S rDNA domain D1/D2 sequences were determined by the direct method and found to differ by three base substitutions. Since both were isolated from the same area, Yaku Island, it could be possible that they descended from a common ancestor. This hypothesis could also be supported by the identity of most physiological characters and chromosome numbers (Table 3, Fig. 2).

S. yakushimaensis and S. humaticus are also close to $S$. transvaalensis, forming a cluster supported at a bootstrap level of $95 \%$ (Fig. 3). In comparison with the $26 \mathrm{~S}$ rDNA domain D1/D2 sequence of $S$. transvaalensis (GenBank accession no. U68549), that of S. humaticus IFO $10673^{\mathrm{T}}$ had three base substitutions and one base deletion and that of S. yakushimaensis IFO $1889^{\mathrm{T}}$ had six base substitutions and one base deletion. Similarities among their 26S rDNA domain D1/ D2 sequences showed that $S$. transvaalensis is closer to S. humaticus IFO $10673^{\mathrm{T}}$ than to $S$. yakushimaensis IFO $1889^{\mathrm{T}}$. This is also supported by DNA-DNA hybridization where $S$. humaticus showed an intermediate relationship to $S$. transvaalensis $(49.7 \%)$, but only $30.5 \%$ to $S$. yakushimaensis (Table 2). This is, however, in contrast with phylogenetic analyses based on rDNA sequences, where $S$. humaticus and $S$. yakushimaensis were closest. One possible explanation for the low DNA-DNA similarities of S. yakushimaensis to both $S$. humaticus and $S$. transvaalensis is that $S$. yakushimaensis, which had differentiated from $S$. humaticus, may have evolved more rapidly, resulting in a high frequency of chromosomal rearrangement and decreasing $\mathrm{G}+\mathrm{C}$ contents in the non-coding regions giving the lowest $\mathrm{G}+\mathrm{C}$ content for strains of Saccharomyces (31.0-31.5 mol\%) (Table 2).

\section{ACKNOWLEDGEMENTS}

We are very grateful to Professor A. Vaughan-Martini for many helpful discussions and critical reading of this manuscript.

\section{REFERENCES}

Banno, I. \& Mikata, K. (1981). Ascomycetous yeasts isolated from forest materials in Japan. Inst Ferment Osaka Res Commun 10, 10-19.

Carle, G. F. \& Olson, M. V. (1984). Separation of chromosomal
DNA molecules from yeast by orthogonal-field-alternation gel electrophoresis. Nucleic Acids Res 12, 5647-5664.

Ezaki, T., Hashimoto, Y., Takeuchi, N., Yamamoto, H., Liu, S.-L., Miura, H., Matsui, K. \& Yabuuchi, E. (1988). Simple genetic method to identify viridans group streptococci by colorimetric dot hybridization and fluorometric hybridization in microdilution wells. J Clin Microbiol 26, 1708-1713.

Ezaki, T., Hashimoto, Y. \& Yabuuchi, E. (1989). Fluorometric deoxyribonucleic acid-deoxyribonucleic acid hybridization in microdilution wells as an alternative to membrane filter hybridization in which radioisotopes are used to determine genetic relatedness among bacterial strains. Int $J$ Syst Bacteriol 39, 224-229.

Felsenstein, J. (1985). Confidence limits on phylogenies: an approach using the bootstrap. Evolution 39, 783-791.

Hisatomi, T., Yanagishima, N. \& Banno, I. (1986). Induction of heterothallic strains and their genetic and physiological characterization in a homothallic strain of the yeast Saccharomyces exiguus. Curr Genet 10, 887-892.

Hisatomi, T., Yanagishima, N., Sakurai, A. \& Kobayashi, H. (1988). Interspecific actions of $\alpha$ mating pheromones on the a matingtype cells of three Saccharomyces yeasts. Curr Genet 13, 25-27.

Holm, C., Meeks-Wagner, D. W., Fangman, W. L. \& Botstein, D. (1986). A rapid, efficient method for isolating DNA from yeast. Gene 42, 169-173.

James, S. A., Collins, M. D. \& Roberts, I. N. (1994). Genetic interrelationship among species of the genus Zygosaccharomyces as revealed by small-subunit rRNA gene sequences. Yeast 10, 871-881.

James, S. A., Cal, J., Roberts, I. N. \& Collins, M. D. (1997). A phylogenetic analysis of the genus Saccharomyces based on 18S rRNA gene sequences: description of Saccharomyces kunashirensis sp. nov. and Saccharomyces martiniae sp. nov. Int J Syst Bacteriol 47, 453-460.

Kaneko, Y. \& Banno, I. (1991). Reexamination of Saccharomyces bayanus strains by DNA-DNA hybridization and electrophoretic karyotyping. Inst Ferment Osaka Res Commun 15, $30-41$.

Kimura, M. (1980). A simple method for estimating evolutionary rates of base substitutions through comparative studies of nucleotide sequences. J Mol Evol 16, 111-120.

Kurtzman, C. P. (1998). Zygosaccharomyces Barker. In The Yeasts, a Taxonomic Study, 4th edn, pp. 424-432. Edited by C. P. Kurtzman \& J. W. Fell. Amsterdam: Elsevier.

Kurtzman, C. P. \& Fell, J. W. (1998). The Yeasts, a Taxonomic Study, 4th edn. Amsterdam: Elsevier.

Kurtzman, C. P. \& Robnett, C. J. (1997). Identification of clinically important ascomycetous yeasts based on nucleotide divergence in the $5^{\prime}$ end of the large-subunit (26S) ribosomal DNA gene. $J$ Clin Microbiol 35, 1216-1223.

Meyen, J. (1838). Jahresbericht über die Resultate der Arbeiten im Felde der physiologischen Botanik von dem Jahre 1837. Archiv für Naturgeschichte 4 (Zweiter Band), 1-186.

Mikata, K. \& Yamada, Y. (1999). The ubiquinone system in Hasegawaea japonica (Yukawa et Maki) Yamada et Banno: a new method for identifying ubiquinone homologs from yeast cells. Inst Ferment Osaka Res Commun 19, 41-46.

Naumov, G. I., Naumova, E. S. \& Korhola, M. (1995). Karyotypic relationships among species of Saccharomyces sensu lato: $S$. castellii, S. dairensis, S. unisporus and S. servazzii. Syst Appl Microbiol 18, 103-108.

Naumova, E. S., Naumov, G. I. \& Korhola, M. (1996). Use of 
molecular karyotyping for differentiation of species in the heterogeneous taxon Saccharomyces exiguus. J Gen Appl Microbiol 42, 307-314.

Reess, M. (1870). Botanische Untersuchungen uber die Alkoholgarhungspilze. Leipzig: Felix.

Saiki, R. K., Gelfand, D. H., Stoffel, S., Scharf, S. J., Higuchi, R., Horn, G. T., Mullis, K. B. \& Erlich, H. A. (1988). Primer-directed enzymatic amplification of DNA with a thermostable DNA polymerase. Science 239, 487-491.

Saitou, N. \& Nei, M. (1987). The neighbor-joining method: a new method for reconstructing phylogenetic trees. Mol Biol Evol 4, 406-425.

Tamaoka, J. \& Komagata, K. (1984). Determination of DNA base composition by reversed-phase high-performance liquid chromatography. FEMS Microbiol Lett 25, 125-128.

Ueda-Nishimura, K. \& Mikata, K. (1999). A new yeast genus, Tetrapisispora gen. nov.: Tetrapisispora iriomotensis sp. nov., Tetrapisispora nanseiensis sp. nov. and Tetrapisispora arboricola sp. nov., from the Nansei Islands, and reclassification of Kluyveromyces phaffii (van der Walt) van der Walt as Tetrapisispora phaffii comb. nov. Int J Syst Bacteriol 49, 1915-1924.

Vaughan-Martini, A. (1995). Saccharomyces barnetti and Sac- charomyces spencerorum: two new species of Saccharomyces sensu lato (van der Walt). Antonie Leeuwenhoek 68, 111-118.

Vaughan-Martini, A. \& Barcaccia, S. (1996). A reconsideration of species related to Saccharomyces dairensis (Naganishi). Int $J$ Syst Bacteriol 46, 313-317.

Vaughan-Martini, A. \& Martini, A. (1998). Saccharomyces Meyen ex Reess. In The Yeasts, a Taxonomic Study, 4th edn, pp. 358-371. Edited by C. P. Kurtzman \& J. W. Fell. Amsterdam: Elsevier.

Vaughan-Martini, A., Martini, A. \& Cardinali, G. (1993). Electrophoretic karyotyping as a taxonomic tool in the genus $\mathrm{Sac}$ charomyces. Antonie Leeuwenhoek 63, 145-156.

Vaughan-Martini, A., Barcaccia, S. \& Pollacci, P. (1996). Saccharomyces rosinii sp. nov., a new species of Saccharomyces sensu lato (van der Walt). Int J Syst Bacteriol 46, 615-618.

Wyder, M. T, Meile, L. \& Teuber, M. (1999). Description of Saccharomyces turicensis sp. nov., a new species from kefyr. Syst Appl Microbiol 22, 420-425.

Yarrow, D. (1998). Methods for the isolation, maintenance and identification of yeasts. In The Yeasts, a Taxonomic Study, 4th edn, pp. 77-100. Edited by C. P. Kurtzman \& J. W. Fell. Amsterdam: Elsevier. 